\title{
Reliability Modeling and Evaluation Method of CNC Grinding Machine Tool
}

\author{
Yongjun Liu ${ }^{1, *}$, Hua Peng ${ }^{2}$ and Yong Yang ${ }^{3}$ \\ 1 School of intelligent manufacturing, Huanghuai University, Zhumadian 463000, China \\ 2 Library of Huanghuai University, Zhumadian 463000, China; penghua1979@sohu.com \\ 3 School of mechanical and energy engineering, Huanghuai University, Zhumadian 463000, China; \\ yy_robsin@126.com \\ * Correspondence: 20151503@huanghuai.edu.cn; Tel.: +86-396-291-9286
}

Received: 25 November 2018; Accepted: 17 December 2018; Published: 21 December 2018

check for updates

Featured Application: The proposed research aims to provide one accurate and reliable evaluation method for the subsystem and system of electromechanical equipment.

\begin{abstract}
How to determine subsystem's reliability and evaluate the whole machine's reliability of the CNC (computer numerical control) grinding machine tool is important for reliability design and promotion. Each subsystem was modeled by a different distribution based on collected data and the models were optimized by the modified gray correlation degree, which was called the similar slope and close median gray correlation method. Methods of generating random data were proposed for different models. The reliability evaluation algorithm based on Monte Carlo simulation was studied and the whole machine's reliability of the CNC grinder was evaluated by the algorithm. The evaluation result by the Monte Carlo algorithm was compared using the observed value and the evaluation value by the whole fitting method. The results show that the point estimation value evaluated by the algorithm based on Monte Carlo has high accuracy. The interval estimation by the Monte Carlo method was $20.2 \%$ and $38.1 \%$ by the observed value and the whole fitting value, respectively, which has more practical engineering significance. The reliability evaluation method can be used to assess the whole CNC grinding machine's reliability in reliability design, and is also one reference for reliability evaluation of other electromechanical equipment.
\end{abstract}

Keywords: reliability modeling; reliability optimization; reliability evaluation; Monte Carlo simulation; CNC grinding machine tool

\section{Introduction}

The CNC (computer numerical control) grinding machine tool is widely used in automotive, aircraft, and other industries to grind precise parts as the last process, and its reliability has an important influence on the processing quality and production efficiency. At present, high-performance CNC machine tools require reliability design. Reliability assessment is an important part of the reliability design. From reliability evaluation, the designer can not only understand the reliability level of the current equipment, they can also find the weak link, as well as test whether or not the reliability design meets the requirements. At the same time, reliability evaluation can select the outsourcing products whose reliability meets the requirements.

There are many reliability evaluation methods and scholars have conducted extensive research on the reliability evaluation of machine tools or similar equipment. The reliability of the CNC machine tool was evaluated using a go-flow methodology by Yu et al. [1]. The reliability of the CNC machine tool was analyzed and evaluated using three parameters of the Weibull distribution by Zhang et al. [2]. 
The reliability of the CNC machine tool was evaluated using the exponential distribution by Dai et al. [3]. The tool reliability of the CNC machine was evaluated using the logistic model by Chen et al. [4]. The reliability of the structural system was reassessed using the Bayesian network by Sankaran et al. [5]. The reliability was analyzed through reliability data and evaluated using the maximum likelihood estimation by David et al. [6]. The reliability of the machining center was evaluated using failure mode analysis by Kim et al. [7]. The reliability of the drilling machine was evaluated using neural network and wavelet analysis by Chinnam et al. [8]. The reliability of typical modular mechanical equipment was evaluated using the Weibull distribution by Neumann et al. [9]. Sung proposed the lifetime estimation method by accelerated degradation test [10]. Cheng et al. used Monte Carlo simulation to analyze the machine tool's machining accuracy reliability [11]. Wei et al. proposed one reliability assessment method for mechanical equipment using the performance-feature model [12]. The cutting tool's reliability was predicted based on acoustic emission and logistic regression model by Hongkun et al. [13]. There are some shortcomings of the current research. (1) The machine is usually regarded as one whole to study on the condition that the reliability data are not enough. (2) The distribution of the whole machine is usually supposed as the exponential distribution or Weibull distribution. In fact, the CNC machine tool is one complex mechanical, electrical, and hydraulic system. Furthermore, the distribution model of each component is not same and the difference is ignored by the above method (signed as the whole fitting method). The pertinence is not strong in reliability design by the whole fitting method. Different subsystems of the CNC grinding machine are designed and manufactured by different enterprises; at this point, subsystem division and studying have more practical significance than the whole fitting method.

In order to evaluate the reliability accurately of CNC grinding machine tools with less field data, one system reliability evaluation method for each subsystem of the CNC machine tool being modeled, as well as then running Monte Carlo simulation, was proposed in the paper, which makes the evaluation scope narrower and means that it has more engineering significance.

\section{Modeling of Subsystem}

\subsection{Reliability Models}

The CNC grinding machine can be divided into different subsystems according to their structure and function, and the distribution types of each subsystem are not same. The fault rate curves of mechanical and electrical products or components are similar to the "bathtub curve", and usually only the first and second paragraphs of the bathtub curve are studied, which are the early failure period and occasional failure period. In statistics, there are many distribution types that are close to the bathtub curve, such as the exponential distribution, Weibull distribution, normal distribution, lognormal distribution, gamma distribution, and so on, as shown in Figure 1. The probability density functions of the above-mentioned five distribution types are shown in Equations (1) to (5). In this paper, five distribution models are used to fit for each subsystem separately, and the optimum model for each subsystem is selected.

$$
\begin{gathered}
f(t)=\lambda e^{-\lambda t} \\
f(t)=\frac{\beta}{\eta}\left(\frac{t-\gamma}{\eta}\right)^{\beta-1} \exp \left(-\left(\frac{t-\gamma}{\eta}\right)^{\beta}\right) \\
f(t)=\frac{1}{\sigma \sqrt{2 \pi}} \exp \left(-\frac{(t-\mu)^{2}}{2 \sigma^{2}}\right) \\
f(t)=\frac{1}{\sigma_{l} t \sqrt{2 \pi}} \exp \left(-\frac{\left(\ln t-\mu_{l}\right)^{2}}{2 \sigma_{l}^{2}}\right) \\
f(t)=\frac{1}{b^{a} \Gamma(a)} t^{a-1} e^{-\frac{t}{b}}
\end{gathered}
$$




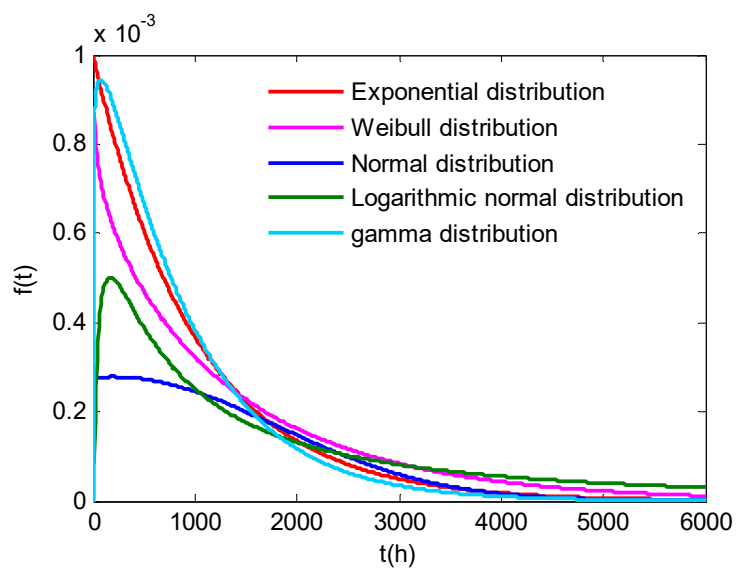

\subsection{Reliability Data Grouping}

Figure 1. Typical probability density curves.

Reliability data are needed for reliability modeling. Reliability data should be classified to each subsystem. The maximal time between failures can be divided equally into $\mathrm{k}$ intervals and the reliability data can be classified into the corresponding intervals for each subsystem. The value of $\mathrm{k}$ can be from 6 to 10. The fault number and probability density can be calculated as shown in Table 1 .

$$
f_{i}=\frac{n_{i}}{\Delta t \sum n_{i}}
$$

Table 1. Reliability data acquiring and grouping.

\begin{tabular}{cccccc}
\hline Group Number & $\boldsymbol{t}_{\boldsymbol{i}-}$ & $\boldsymbol{t}_{\boldsymbol{i}}$ & $\boldsymbol{t}_{\boldsymbol{i +}}$ & $\boldsymbol{n}_{\boldsymbol{i}}$ & $f_{\boldsymbol{i}}$ \\
\hline 1 & 0 & $t_{\mathrm{m}} / 2 k$ & $t_{\mathrm{m}} / k$ & $n_{1}$ & $f_{1}$ \\
2 & $t_{\mathrm{m}} / k$ & $3 t_{\mathrm{m}} / 2 k$ & $2 t_{\mathrm{m}} / k$ & $n_{2}$ & $f_{2}$ \\
$\ldots$ & $\ldots$ & $\ldots$ & $\ldots$ & $\ldots$ & $\ldots$ \\
$\mathrm{k}$ & $(k-1) \cdot t_{\mathrm{m}} / k$ & $(2 k-1) \cdot t_{\mathrm{m}} / 2 k$ & $t_{\mathrm{m}}$ & $n_{\mathrm{k}}$ & $f_{\mathrm{k}}$ \\
\hline
\end{tabular}

Note: $t_{\mathrm{m}}$ : the maximal time between failures; $t_{i-}$ : the left end of the $i$ th time interval; $t_{i+}$ : the right end of the $i$ th time interval; $t_{i}$ : the median of the $i$ th time interval; $n_{i}$ : the fault number of the $i$ th time interval; $f_{i}$ : the probability density of the $i$ th time interval, which can be calculated by Equation (6). In Equation (6), $\Delta t$ is the time interval length.

\subsection{Probability Density Function Fitting}

After the probability density of each interval is calculated, the $k$ group data pair can be obtained as $\left(t_{i}, f_{\mathrm{i}}\right)$. The probability density function can be fitted according to the data pair using the least squares method or maximum likelihood method for each subsystem by different distribution type, and the point estimation and interval estimation of the parameters of the probability density function can be executed [14].

\subsubsection{Exponential Distribution}

The accumulative distribution function of the exponential distribution is shown in Equation (7) and has one parameter, $\lambda$.

$$
F\left(t_{i}\right)=1-e^{-\lambda t_{i}}
$$

Taking the logarithm on both sides of Equation (7), the linear equation can be created. If the sample data meet exponential distribution, $t_{i}$ and $\ln \left[1-F\left(t_{i}\right)\right]$ are linear and the observed value of parameters $\lambda$ can be calculated by Equation (8).

$$
\hat{\lambda}=-a
$$

\subsubsection{Weibull Distribution}

The accumulative distribution function of the two-parameter Weibull distribution is shown in Equation (9) and has two parameters, $m$ and $\eta$. 


$$
F\left(t_{i}\right)=1-\exp \left[-\left(t_{i} / \eta\right)^{m}\right]
$$

Taking the logarithm twice on both sides of Equation (9) and supposing $y=\ln \ln \left(1 /\left(1-F\left(t_{i}\right)\right)\right)$ and $x=\ln t_{i}$, the linear equation can be created. If the sample data meet the Weibull distribution, $y=\ln \ln \left(1 /\left(1-F\left(t_{i}\right)\right)\right)$ and $\ln t_{\mathrm{i}}$ are linear and the observed value of parameters $m$ and $\eta$ can be calculated by Equation (10).

$$
\left\{\begin{array}{c}
\hat{m}=b \\
\hat{\eta}=\exp \left(-\frac{a}{b}\right)
\end{array}\right.
$$

\subsubsection{Normal Distribution}

The probability density function of the normal distribution has two parameters, $\mu$ and $\sigma$. The accumulative distribution function meets the relation shown in Equation (11) if the function is converted to standard normal distribution.

$$
F\left(t_{i}\right)=\Phi\left(\frac{t_{i}-\mu}{\sigma}\right)=\Phi\left(z_{i}\right)
$$

The linear equation can be constructed for the normal distribution as shown in Equation (12).

$$
t_{i}=\sigma z_{i}+\mu
$$

In Equations (11) and (12), $z_{i}$ is the lower fractile of standard normal distribution and can be calculated by the inverse function of the standard normal distribution. If the sample data meet normal distribution, $z_{i}$ and $t_{i}$ are linear and the observed value of parameters $\mu$ and $\sigma$ can be calculated by Equation (13).

$$
\left\{\begin{array}{l}
\hat{\mu}=b \\
\hat{\sigma}=a
\end{array}\right.
$$

\subsubsection{Logarithmic Normal Distribution}

The probability density function of the logarithmic normal distribution has two parameters, $\mu$ and $\sigma$. The accumulative distribution function meets the relation as shown in Equation (14) if the function is converted to standard normal distribution.

$$
F\left(\ln t_{i}\right)=\Phi\left(\frac{\ln t_{i}-\mu}{\sigma}\right)=\Phi\left(z_{i}\right)
$$

The linear equation can be constructed for the logarithmic normal distribution as shown in Equation (15).

$$
\ln t_{i}=\sigma z_{i}+\mu
$$

If the sample data meet the logarithmic normal distribution, $z_{i}$ and $\ln t_{i}$ are linear and the observed value of parameters $\mu$ and $\sigma$ can be calculated by Equation (16).

$$
\left\{\begin{array}{l}
\hat{\mu}=b \\
\hat{\sigma}=a
\end{array}\right.
$$

\subsubsection{Gamma Distribution}

The probability density function of the gamma distribution cannot be constructed as one linear equation, and the parameters $\alpha$ and $\beta$ can be calculated by the maximum likelihood method. If the data meet the gamma distribution, the likelihood equation can be constructed in Equation (17). 


$$
L\left(t_{1}, t_{2}, \cdots, t_{k}, \alpha, \beta\right)=\prod_{i=1}^{k} \frac{\beta^{\alpha} t_{i}^{\alpha-1} e^{-\beta t_{i}}}{\Gamma(\alpha)}
$$

The execute logarithm of Equations (17) and (18) can be gained. In Equation (18), $\alpha$ and $\beta$ can be solved by the numerical iteration method.

$$
\left\{\begin{array}{c}
\frac{k d(\ln \Gamma(\alpha))}{d t}-k \ln \beta-\sum \ln t_{i}=0 \\
k \alpha \beta-\beta^{2} \sum \ln t_{i}=0
\end{array}\right.
$$

\subsection{Model Optimization}

The reliability assessment results will differ greatly if the reliability models are different. Each subsystem will be fitted by five distribution functions, so that it is necessary to select one optimal model as the reliability model for each subsystem. The $K-S$ hypothesis test method, correlation coefficient method, and gray correlation degree method are the normal model optimization methods $[15,16]$.

The accuracy of the $K-S$ hypothesis test is proportional to the number of reliability data. The correlation coefficient method is used to optimize the models that are linear or can be converted into a linear relationship. The standard gray correlation is mainly to judge the similarity of two models $[17,18]$. Not only will the similarity be judged, but also the closeness should be calculated between sample data and the fitted model when the model optimization is executed. One modified gray correlation model optimization method is proposed based on the standard gray correlation, which is called the similar slope and close median gray correlation. The basic idea of the model optimization method is to judge the similarity degree of the slope of adjacent points between the sample data and fitted curve and the close degree of the median between the sample data and fitted curve. The algorithm of the model optimization method is shown as follows and the flow chart is shown in Figure 2.

1. Calculate the adjacent points' slope of sample data and fitted curve, as shown in Equation (19).

$$
\left\{\begin{aligned}
K_{i} & =\frac{\hat{F}\left(t_{i+1}\right)-\hat{F}\left(t_{i}\right)}{t_{i+1}-t_{i}} \\
K_{j i} & =\frac{F_{j}\left(t_{i+1}\right)-F_{j}\left(t_{i}\right)}{t_{i+1}-t_{i}}
\end{aligned}\right.
$$

where

$\hat{F}\left(t_{i}\right)$ is the function value of the $i$ th sample data,

$K_{j i}$ is the slope of the $i$ th and $(i+1)$ th sample data of the $j$ th fitting function, and

$F_{j}\left(t_{i}\right)$ is the function value of the $j$ th fitting function when the independent variable equals to the value of the $i$ th sample data.

2. Calculate the adjacent points' median of sample data and fitted curve, as shown in Equation (20).

$$
\left\{\begin{aligned}
V_{i} & =\frac{\hat{F}\left(t_{i+1}\right)+\hat{F}\left(t_{i}\right)}{2} \\
V_{j i} & =\frac{F_{j}\left(t_{i+1}\right)+F_{j}\left(t_{i}\right)}{2}
\end{aligned}\right.
$$

3. Calculate the absolute value of difference of slope and median, as shown in Equation (21).

$$
\left\{\begin{array}{l}
D K_{j i}=\left|K_{i}-K_{j i}\right| \\
D V_{j i}=\left|V_{i}-V_{j i}\right|
\end{array}\right.
$$


4. Maximum image processing of slope and median, as shown in Equation (22).

$$
\left\{\begin{aligned}
R K_{j i} & =D K_{j i} / \max \left(D K_{j i}\right) \\
R V_{j i} & =D V_{j i} / \max \left(D V_{j i}\right)
\end{aligned}\right.
$$

5. Calculate the gray correlation between sample data and fitted function, as shown in Equation (23).

$$
R_{k v j}=\frac{1}{n-1} \sum_{i=1}^{n-1} R K_{j i} \frac{1}{n-1} \sum_{i=1}^{n-1} R V_{j i}
$$

6. Normalization. In Equation (23), $R_{k v j}$ is the similarity and closeness between the $j$ th fitted curve and the sample data. The smaller the value of $R_{k v j}$, the better the fitting. Usually, the big gray correlation degree indicates the good fitting, and its value is between 0 and 1 , which needs to be normalized, as shown in Equation (24).

$$
R_{g j}=\frac{\min \left(R_{k v j}\right)}{R_{k v j}+\rho \min \left(R_{k v j}\right)}
$$

In Equation (24), $\rho$ is the discrimination adjustment coefficient, which is between $[0,1]$. The smaller $\rho$ is, the greater the degree of distinction will be. The gray correlation can be calculated using the five distribution functions. The distribution function of the maximum value of the gray correlation will be selected as the reliability model of the corresponding subsystem.

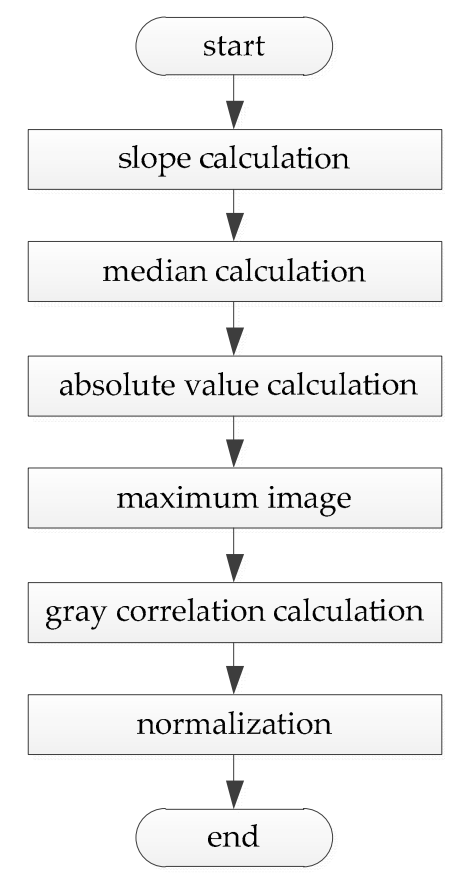

Figure 2. Flow char of model optimization method.

\section{Reliability Evaluation Method}

Although the reliability model of each subsystem of the $\mathrm{CNC}$ grinding machine tool can be done using the method in Section 2, the reliability of each subsystem can be evaluated by the model and then the system reliability can be gained by the subsystem's reliability. In fact, the time when each subsystem breaks down is random and the MTBF (mean time between failures) of each subsystem also changes. The Monte Carlo method can simulate the randomness well, thus the Monte Carlo simulation is used to evaluate the whole machine's reliability of the CNC grinding machine tool in this paper. 


\subsection{Monte Carlo Simulation}

Monte Carlo is also known as the statistical test method, stochastic simulation method, and random sampling method, which is one method for studying distribution characteristics by repeatedly generating time series to calculate the estimated parameters and statistics $[19,20]$.

The frequency of events or arithmetic mean of random variable $X$ is regarded as the solution of the problem by the Monte Carlo simulation method. Suppose that the samples of the random variable $X$ are as follows: $X_{1}, X_{2}, \ldots, X_{N}$, the arithmetic mean of the random variable $X$ is shown in Equation (25).

$$
\bar{X}=\frac{1}{N} \sum_{i=1}^{N} X_{i}
$$

Equation (26) can be gained by the strong law of large number, in which the $\varepsilon$ is any small positive. From Equation (26), when $N$ is big enough, $\bar{X}$ converges to $E(X)$, which is the convergence guarantee of the Monte Carlo simulation method [21,22].

$$
\lim _{N \rightarrow \infty} P\{|\bar{X}-E(X)|<\varepsilon\}=1
$$

Equation (27) can be gained by the central limit theorem, in which the $\mu_{\alpha}$ is any value.

$$
P\left\{|\bar{X}-E(X)|<\frac{\mu_{\alpha} \sigma}{\sqrt{N}}\right\} \approx \frac{2}{\sqrt{2 \pi}} \int_{0}^{u_{\alpha}} \exp \left(t^{2} / 2\right) d t=1-\alpha
$$

where

$\alpha$ is the significant level,

$\sigma$ is the standard deviation of the random variable $X$, and

$\mu_{\alpha}$ is the quantile of the standard normal distribution.

It is necessary to construct one probability statistics model, define the random variable, sample based on the known distribution model, generate random data, and calculate when using Monte Carlo method. The accuracy of the Monte Carlo method is related to the number of samples-the larger the number of samples, the smaller the error [23].

\subsection{Random Data Generation}

The distribution of time between failures of each subsystem of the CNC grinding machine tool may meet the exponential distribution, Weibull distribution, normal distribution, lognormal distribution, or gamma distribution. The random data generation method is put forward as below.

If the distribution function of random variable $X$ is $F(x)$, which is continuous, $F(x)$ is a uniform distribution of random variables on the [0,1]. In order to obtain the random data that meet distribution function of $F(x)$, the random data can be solved by the uniform distribution of $r$ on the [0,1], as shown in Equation (28).

$$
r=F(x)
$$

In Equation (28), $x$ is the random data of distribution function $F(x)$. The random data that meet the exponential distribution, Weibull distribution, normal distribution, lognormal distribution, and gamma distribution can be calculated by Equation (28), as shown in Equations (29) to (33).

$$
\begin{gathered}
x=-\frac{1}{\lambda} \ln r \\
x=\eta(-\ln r)^{1 / \beta} \\
x=\mu+\sigma \sqrt{-2 \ln r_{1}} \cos \left(2 \pi r_{2}\right)
\end{gathered}
$$




$$
\begin{gathered}
x=e^{\mu+\sigma \sqrt{-2 \ln r_{1}} \cos \left(2 \pi r_{2}\right)} \\
x=-b \ln \left(\prod_{i=1}^{\text {round }(a)} r_{i}\right)
\end{gathered}
$$

\subsection{Reliability Evaluation Algorithm}

\section{Work Time Calculation}

Assuming the average life cycle of the CNC grinding machine tool is $T_{L}$ years, the average annual maintenance time of the life cycle is $T_{M}$, the annual average normal downtime is $T_{S}$, and then the normal working time in the life cycle of the CNC grinding machine $T_{W}$ is as shown in Equation (34).

$$
T_{W}=\left(365 \times 24-T_{M}-T_{S}\right) \times T_{L}
$$

\section{Quantity of Random Data Calculation}

The quantity of generated random data of time between failures of each subsystem of each CNC grinder can be calculated by Equation (35). In Equation (35), $i$ is the number of the subsystem ( $i=1,2$, $\ldots, n), n$ is the amount of the subsystem, and $M T B F_{i}$ is the MTBF of the $i$ th subsystem.

$$
k_{i}=\frac{T_{W}}{M T B F_{i}}
$$

\section{Dimension of Random Data Calculation}

To enhance the accuracy, $m$ CNC grinders are selected for simulation. The dimension of random data $d_{i}$ generated by the $i$ th subsystem can be calculated by Equation (36).

$$
d_{i}=m \times k_{i}
$$

\section{Random Data Generation}

After calculating the distribution type and parameters of distribution function of each subsystem, the random data of time between failures of each subsystem can be generated according to Equations (29) to (33). The random data matrix $\boldsymbol{T}_{i}$ of the $i$ th subsystem is shown in Equation (37).

$$
\boldsymbol{T}_{i}=\left[\begin{array}{cccc}
T_{00}^{i} & T_{01}^{i} & \cdots & T_{0 k_{i}}^{i} \\
T_{10}^{i} & T_{11}^{i} & \cdots & T_{1 k_{i}}^{i} \\
\vdots & \vdots & \ddots & \vdots \\
T_{m 0}^{i} & T_{m 1}^{i} & \cdots & T_{m k_{i}}^{i}
\end{array}\right]_{\left(m \times k_{i}\right)}
$$

\section{Sum of Every Time Between Failures}

After all subsystems' random data are generated, the sum of every time between failures can be calculated by Equation (38).

$$
T_{\text {sum }}=\sum_{i=1}^{n} \sum_{u=1}^{m} \sum_{v=1}^{k_{i}} T_{u v}^{i}
$$

where $k_{i}$ is the amount of random data of the $i$ th subsystem.

\section{Sum of Fault Amount Calculation}

It is necessary to calculate the sum of fault amount of each subsystem and each CNC grinding machine in the life cycle, as shown in Equation (39). 


$$
n_{\text {sum }}=m \sum_{i=1}^{n} k_{i}
$$

\section{System MTBF Calculation}

The system reliability of $M T B F_{s}^{m}$ can be calculated by the Monte Carlo simulation method, as shown in Equation (40).

$$
\operatorname{MTBF}_{s}^{m}=\frac{T_{\text {sum }}}{n_{\text {sum }}}=\frac{\sum_{i=1}^{n} \sum_{u=1}^{m} \sum_{v=1}^{k_{i}} T_{u v}^{i}}{m \sum_{j=1}^{n} k_{j}}
$$

Taking into account the monotonicity of different distribution functions, the interval estimation of system reliability of the CNC grinding machine can be calculated by replacing the point estimation value with the upper and lower bound of interval estimate value to generate random data in step 4 .

\section{Case Study}

In order to illustrate the application of the reliability model optimization of the subsystems and the Monte Carlo reliability evaluation method, a CNC cylindrical grinding machine is used as a research case. The CNC cylindrical grinding machine is used to grind the shaft parts. The feed precision is $0.001 \mathrm{~mm}$, the roundness of the ground parts is $0.002 \mathrm{~mm}$, and the grinding line speed is $60 \mathrm{~m} / \mathrm{s}$ of the studied CNC cylindrical grinding machine. The CNC cylindrical grinding machine can be divided into 10 subsystems according to their structural characteristics, as shown in Table 2.

\subsection{Modeling of Subsystem}

Fifteen CNC grinding machines were tracked for three years and nine months and 748 failure data were collected. The distribution type and probability density function are solved by data grouping, model optimization by five distribution functions of each subsystem, as shown in Table 2 and the cumulative distribution curve of each subsystem is shown in Figure 3.

\subsection{System Reliability Evaluation}

In order to analyze and compare easily, the system reliability evaluated by the observed value and by the whole fitting method is also listed below.

Table 2. Reliability models and parameters of subsystems. CNC-computer numerical control; MTBF-mean time between failures.

\begin{tabular}{ccc}
\hline Subsystem & Distribution Type & Parameters \\
\hline base & exponential & $\lambda=6.61 \times 10^{-5}$ \\
CNC & gamma & $\mathrm{a}=6.81 \times 10^{-1}, \mathrm{~b}=9.41 \times 10^{3}$ \\
spindle & gamma & $a=7.81 \times 10^{-1}, b=9.68 \times 10^{3}$ \\
feeder & gamma & $a=1.539, b=1.31 \times 10^{3}$ \\
electrical & Weibull & $\beta=3.84 \times 10^{-1}, \eta=6.58 \times 10^{2}$ \\
hydraulic & gamma & $a=7.57 \times 10^{-1}, b=1.36 \times 10^{4}$ \\
cooler & lognormal & $\mu_{l}=7.011, \sigma_{l}=1.644$ \\
lubrication & gamma & $a=6.05 \times 10^{-1}, b=1.26 \times 10^{4}$ \\
header & Weibull & $\beta=5.62 \times 10^{-1}, \eta=1.65 \times 10^{3}$ \\
gauge & gamma & $a=8.44 \times 10^{-1}, b=9.25 \times 10^{3}$ \\
\hline
\end{tabular}




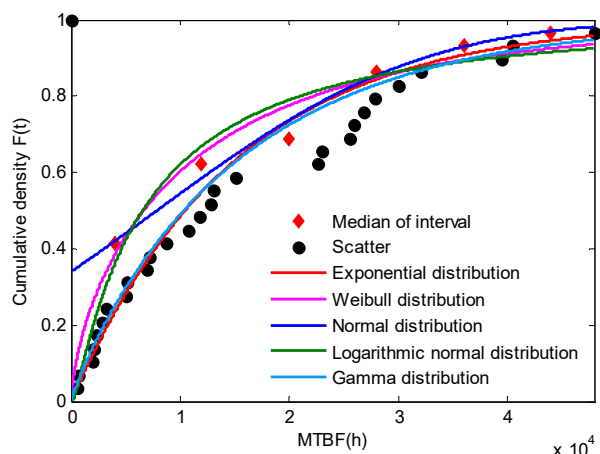

(a) base

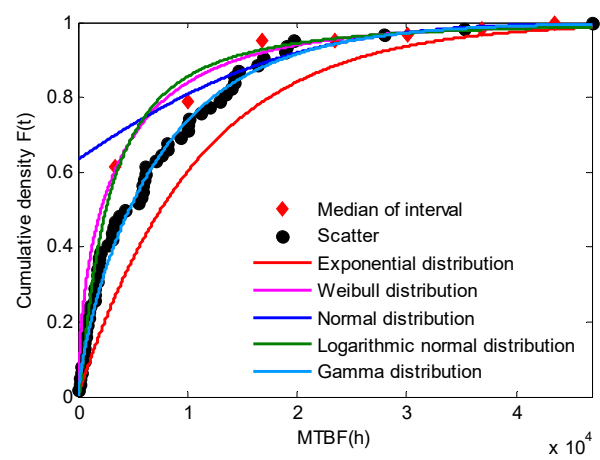

(c) spindle

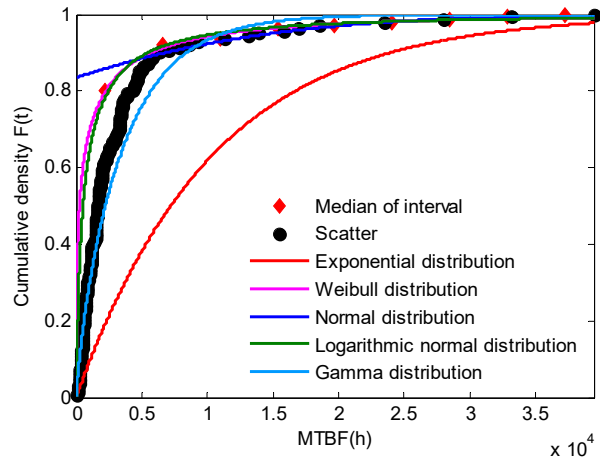

(e) electrical

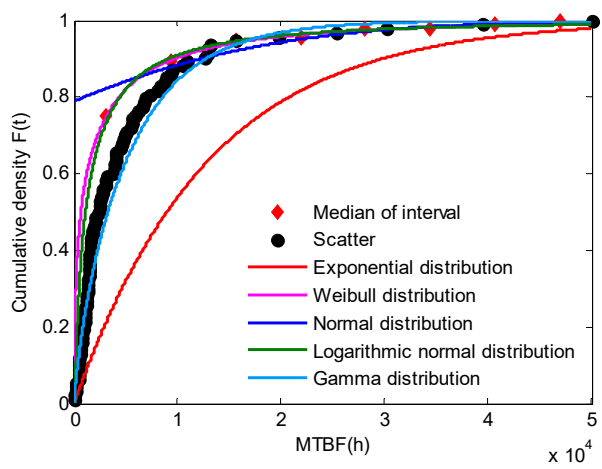

(g) cooler

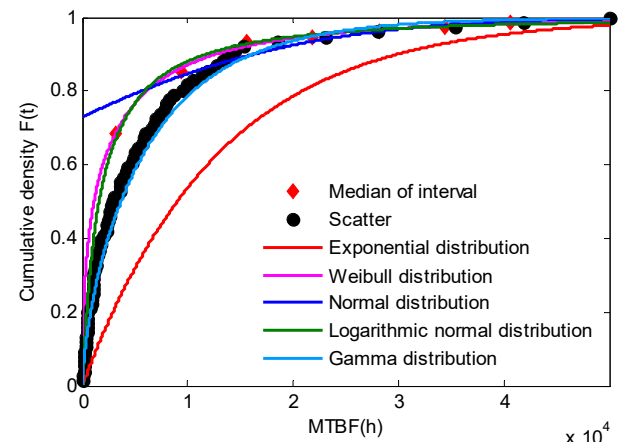

(b) $\mathrm{CNC}$

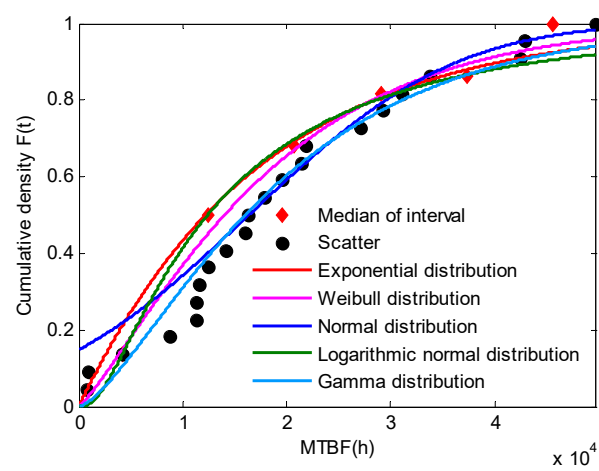

(d) feeder

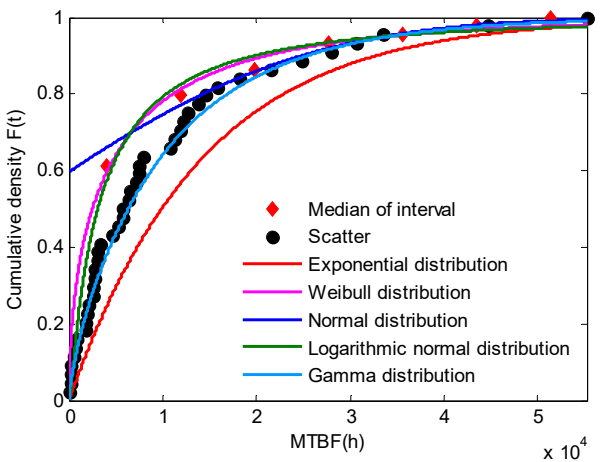

(f) hydraulic

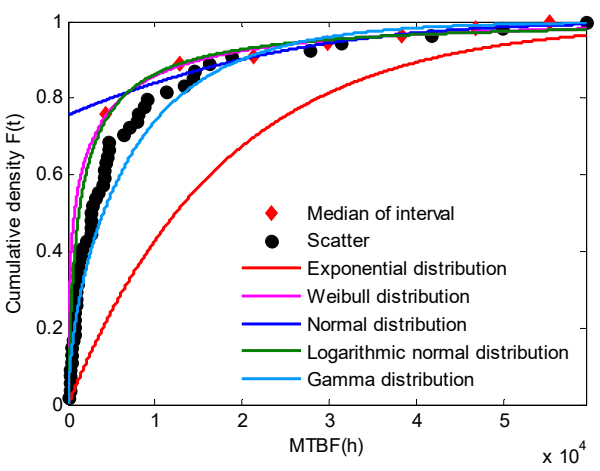

(h) lubrication

Figure 3. Cont. 


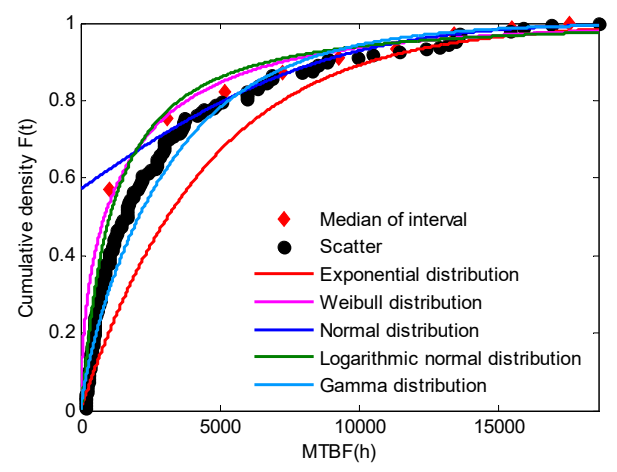

(i) header

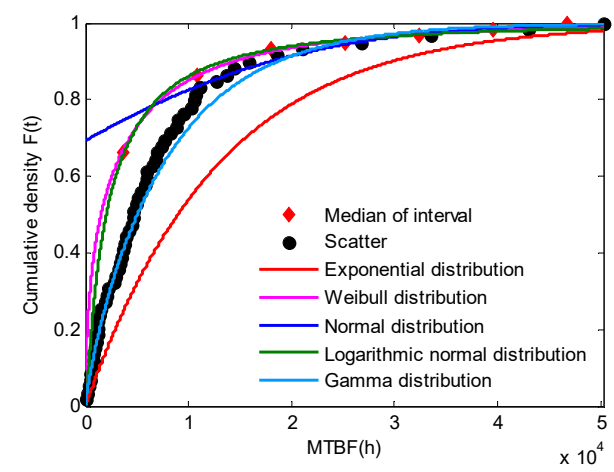

(j) gauge

Figure 3. Cumulative distribution curve of each subsystem. $\mathrm{CNC}$-computer numerical control; MTBF-mean time between failures. (a) base; (b) CNC; (c) spindle; (d) feeder; (e) electrical; (f) hydraulic; (g) cooler; (h) lubrication; (i) header; (j) gauge.

\subsubsection{Observed Value}

- Point estimation

Suppose that the observed value of system reliability is $\mathrm{MTBF}_{s}{ }^{\circ}$, the amount of $\mathrm{CNC}$ grinding machine is $n_{m}$, the year of failure data collecting is $y_{r}$, the day of failure data collecting each year is $d_{r}$, the work time each day is $h_{r}$, the amount of failures is $n_{f}$, and the mean maintenance time is $t_{m a}$, the observed value of system reliability can be calculated by Equation (41).

$$
\operatorname{MTBF}_{s}^{o}=\frac{n_{m} \cdot y_{r} \cdot d_{r} \cdot h_{r}-n_{f} \cdot t_{m a}}{n_{f}}
$$

The observed value of system reliability is $563 \mathrm{~h}$ on condition that the work time each day is $24 \mathrm{~h}$ and the mean maintenance time is $2 \mathrm{~h}$.

- Interval estimation

The interval estimation of system reliability of the CNC grinding machine tool can be calculated by the confidence coefficient, as shown in Equation (42).

$$
\left[\mathrm{MTBF}_{s}{ }^{0} \cdot \mathrm{C}_{L}, \mathrm{MTBF}_{\mathrm{s}}{ }^{0} \cdot \mathrm{C}_{U}\right]
$$

where

$M T B F_{S}{ }^{o}$ is the observed value of system reliability of the CNC grinding machine,

$C_{L}$ is the lower confidence coefficient, and

$C_{U}$ is the upper confidence coefficient.

The interval estimation of system reliability of the CNC grinding machine can be calculated by the confidence coefficient table and the interval is $[415,782] \mathrm{h}$.

\subsubsection{Whole Fitting Method}

At present, many scholars regard the CNC grinding machine as one whole, and the system reliability is evaluated directly by all the failure data. It is one acceptable reliability evaluation method if the failure data are not enough. For comparison, the system reliability is also evaluated by the whole fitting method.

If the CNC grinding machine tool is regarded as a whole, all the failure data belong to the whole grinding machine, and the probability density function of the whole machine can be fitted by the 
method in Section 2, as shown below. Its probability density curve and cumulative distribution curve are shown in Figures 4 and 5, respectively. The distribution type of the system reliability model of the CNC grinding machine is gamma distribution and the parameter $a$ is 0.663 and $b$ is 689.8 .

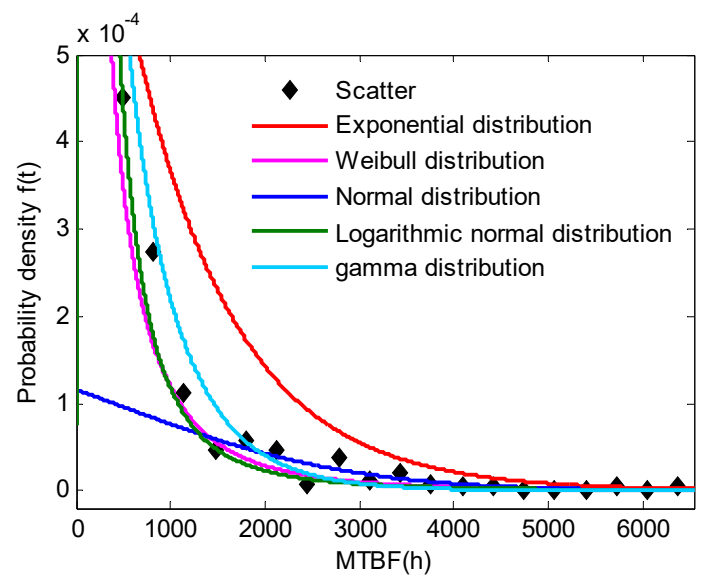

Figure 4. Probability density curve of time between failures of total machine. MTBF-mean time between failures.

The MTBF of the gamma distribution can be solved by its probability density function, as shown in Equation (43). The system reliability of the CNC grinding machine can be calculated, and its point estimation is $457 \mathrm{~h}$ and interval estimation is $[370,564] \mathrm{h}$.

$$
\operatorname{MTBF}_{g}=\int_{0}^{\infty} t f_{g}(t) d t=a b
$$

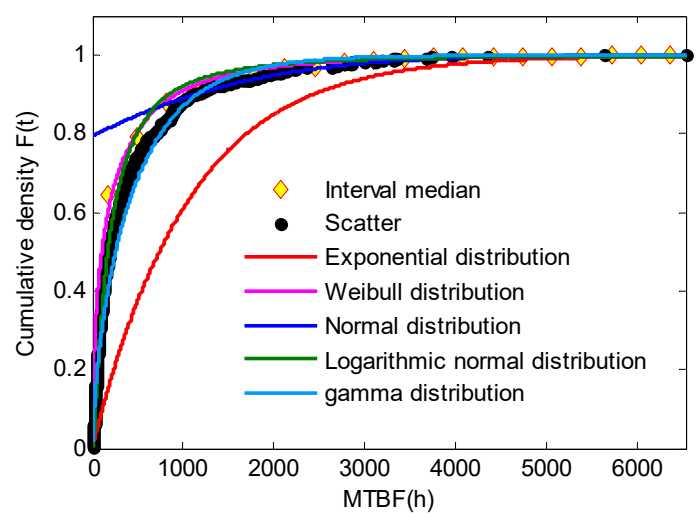

Figure 5. Cumulative distribution curve of time between failures of whole machine.

\subsubsection{Monte Carlo Simulation}

The accuracy of the system reliability evaluation of the Monte Carlo simulation is determined by the simulation time and amount of CNC grinding machine. The evaluation result of different simulation times and different amounts of CNC grinding machine by the algorithm mentioned in Section 2 is shown in Figure 6.

The result will fluctuate severely from that in Figure 6 when the simulation time and amount of the $\mathrm{CNC}$ grinding machine are small, and it will tend to be stable when the simulation time is more than 10 years and the amount of the CNC grinding machine is more than 10, which fluctuates slightly between 530 and $560 \mathrm{~h}$. 


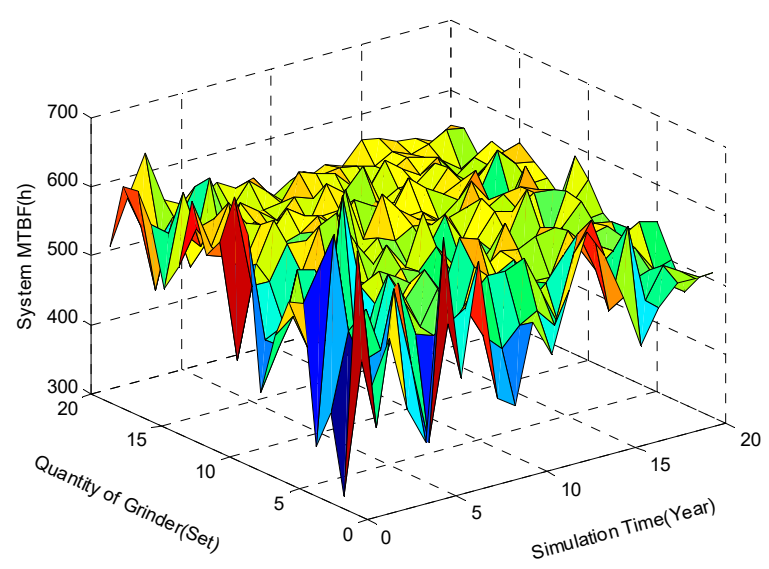

Figure 6. Point estimation comparison of different time and quantity of the computer numerical control $(\mathrm{CNC})$ grinder.

The point estimation of system reliability is $551 \mathrm{~h}$ on condition that the simulation time is 20 years, the amount of the CNC grinding machine is 20, and the interval estimation is between $511 \mathrm{~h}$ and $585 \mathrm{~h}$.

$$
M T B F_{s}^{m k}=\frac{T_{\text {sum }}}{n_{\text {sum }}}=551
$$

\subsection{Comparison and Analysis}

The results evaluated by the three methods are showed in Figure 7, on condition that the confidence level is $95 \%$.

From Figure 7, it can be found that if all the fault data are regarded as a whole to fit, the point estimation of MTBF will be less than MTBF of the observed value. The main reason is that the failure data are too focused on the early time of the reliability test. The evaluation result by the Monte Carlo method is close to the observed value. The interval estimation of Monte Carlo is the least of the three methods, that is to say that the evaluation result is in one smaller interval below a certain confidence level than the other two methods. The smaller the interval estimation range, the bigger the reference to engineering. It can be concluded that the accuracy of the reliability evaluation of the Monte Carlo method is high and it has important reference engineering significance to the reliability design.

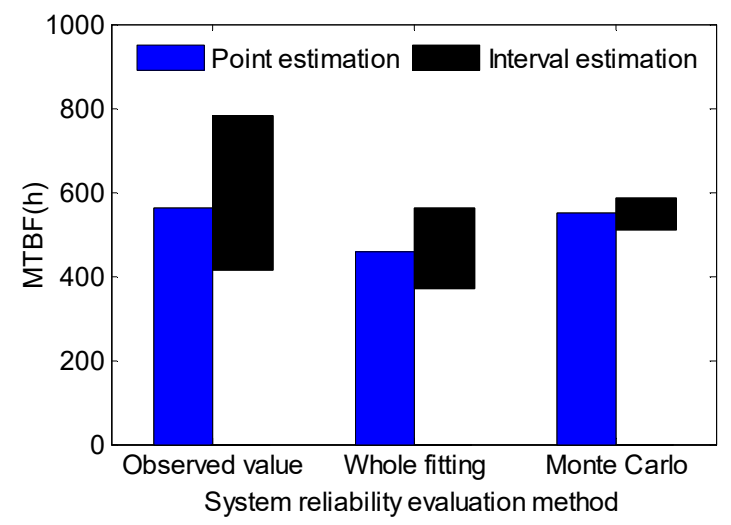

Figure 7. Point estimation and interval estimation of the different methods.

\section{Conclusions}

This paper presented the reliability evaluation method of complex electromechanical products, and illustrated the application of the method to the CNC cylindrical grinding machine. This work led to the following conclusions. 
The traditional method of fitting the whole machine and then carrying out the reliability evaluation can not reveal the failure law of each subsystem. The purpose of the reliability evaluation is to improve the reliability, and the system reliability enhancement is determined by the subsystems' reliability. Compared with the whole machine fitting, the reliability modeling of subsystems has more engineering significance. The reliability modeling and optimization methods of subsystems are proposed by the commonly distribution functions.

The failure time of each subsystem does not strictly follow the reliability model, rather it is random, and Monte Carlo can simulate this randomness very well. The paper proposed one system reliability evaluation method based on Monte Carlo simulation; using this method to evaluate the system reliability is closer to reality.

Through comparative analysis, it is found that the length of the reliability estimation interval evaluated by the Monte Carlo simulation method is $8.2 \%$ and $15.5 \%$ of that evaluated directly by the field data and evaluated by the whole machine evaluation method, respectively. For engineering, the shorter the interval length, the more practical it is.

The system reliability evaluation method of subsystem reliability modeling and Monte Carlo simulation can also be extended to other complex electromechanical equipment when the field data are limited.

Author Contributions: Conceptualization and methodology, Y.L.; validation, Y.Y.; data curation, H.P.; writing—original draft preparation, Y.L. and Y.Y.; writing—review and editing, Y.L.; supervision, Y.L.

Funding: This research was funded by the Key Scientific Research Projects of Henan Higher Education Institutions, grant number 17B460007, and the Science and Technology Research Program of Henan Province, grant number 182102310045 .

Conflicts of Interest: The authors declare no conflict of interest.

\section{References}

1. Yu, J.; Yu, S.; Song, Y.M.; Wang, T.T.; Li, Q.C.; Sun, L.D.; Li, Z.G. Application of Go-Flow Methodology to the Reliability Evaluation in CNC Machine Tools. Adv. Mater. Res. 2013, 694, 1833-1837. [CrossRef]

2. Zhang, Y.Z.; Shen, G.X.; Wu, S. 3-parameter Weibull Distribution for Random Truncated NC Machine Tool Fault Data. J. Jilin Univ. (Eng. Technol. Ed.) 2009, 39, 378-381.

3. Dai, Y. Optimum Plan of Reliability Evaluation of NC Machine Tools Based on Exponential Distribution. China Mech. Eng. 2011, 22, 2735-2738.

4. Chen, B.J; Chen, X.F.; Li, B. Reliability Estimation for Cutting Tool Based on Logistic Regression Model. J. Mech. Eng. 2011, 47, 158-164. [CrossRef]

5. Sankaran, M.; Ruoxue, Z.; Natasha, S. Bayesian networks for system reliability reassessment. Struct. Saf. 2013, 23, 231-251.

6. David, G.G.; Mario, C.S.; Rolando, P.A. Fuzzy Reliability Analysis with Only Censored Data. Eng. Appl. Artif. Intell. 2014, 32, 151-159.

7. Kim, B.S.; Kim, J.S.; Lee, S.H. A study on failure mode analysis of machining centers. J. Korean Soc. Precis. Eng. 2001, 18, 74-79.

8. Chinnam, R.B.; Mohan, P. Online Reliability Estimation of Physical Systems Using Neural Networks and Wavelets. Int. J. Smart Eng. Syst. Des. 2002, 4, 253-264. [CrossRef]

9. Neumann, S.; Woll, L.; Feldermann, A. Modular System Modeling for Quantitative Reliability Evaluation of Technical Systems. Model. Identif. Control 2016, 37, 19-29. [CrossRef]

10. Sung, K.K.; Heonsang, L.; Si-Il, S.; Yong, S.K. Planning of Partially Accelerated Degradation Test with Two Stress Variables for Practical Lifetime Estimation. Appl. Sci. 2018, 8, 2162.

11. Cheng, Q.; Zhao, H.; Zhao, Y. Machining accuracy reliability analysis of multi-axis machine tool based on Monte Carlo simulation. J. Intell. Manuf. 2015, 29, 191-209. [CrossRef]

12. Dai, W.; Chi, Y.; Lu, Z.; Wang, M.; Zhao, Y. Research on Reliability Assessment of Mechanical Equipment Based on the Performance-Feature Model. Appl. Sci. 2018, 8, 1619. [CrossRef]

13. Li, H.; Wang, Y.; Zhao, P.; Zhang, X.; Zhou, P. Cutting tool operational reliability prediction based on acoustic emission and logistic regression model. J. Intell. Manuf. 2015, 26, 923-931. [CrossRef] 
14. Liu, Y.J.; Fan, J.W.; Li, Y. One System Reliability Assessment Method for CNC Grinder. Eksploatacja $i$ Niezawodnosc Maintentance Reliab. 2014, 16, 97-104.

15. Wang, X.Y.; Wan, Z.H. Using Kolmogorov-Smirnov Test on Diagnosis of Gear Faults under Fluctuating Load Conditions. China Mech. Eng. 2009, 20, 1048-1052.

16. Deng, Z.L.; Lin, W.L.; Li, N. The uncertainty entropy of low rate speech quality evaluation and the analyses of the gray correlation. Electron. Express 2015, 12, 1019.

17. Huang, Y.; Chen, G.X.; Chen, Q.F. A Novel Approach of Edge Detection Based on Gray Correlation Degree and Kirsch Operator. Appl. Mech. Mater. 2015, 731, 169-172. [CrossRef]

18. Sun, P. Analysis and Application of Gray Correlation Method. Math. Pract. Theory. 2014, 44, 97-101.

19. Doucet, A.; Pitt, M.K.; Deligiannidis, G. Efficient implementation of Markov chain Monte Carlo when using an unbiased likelihood estimator. Biometrika 2016, 103, 701-717. [CrossRef]

20. Juan, A.; Martinez, V.; Gerardo, G. Reliability Analysis of Distribution Systems with Photovoltaic Generation Using a Power Flow Simulator and a Parallel Monte Carlo Approach. Energies 2016, 9, 537.

21. Singer, D.A. Some Suggested Future Directions of Quantitative Resources Assessment. J. China Univ. Geosci. 2001, 12, 40-44.

22. Bernt, J.L.; Arvid, N.; Ole, E.B. Reliability analysis of corroding pipelines by enhanced Monte Carlo simulation. Int. J. Press. Vessels Piping 2016, 144, 11-17.

23. Jeang, A.; Chen, T.K.; Hwan, C.L. A Statistical Dimension and Tolerance Design for Mechanical Assembly under Thermal Impact. Int. J. Adv. Manuf. Technol. 2002, 20, 907-915. [CrossRef]

(C) 2018 by the authors. Licensee MDPI, Basel, Switzerland. This article is an open access article distributed under the terms and conditions of the Creative Commons Attribution (CC BY) license (http://creativecommons.org/licenses/by/4.0/). 